\title{
Uso de sugammadex en un paciente con insuficiencia renal crónica
}

\author{
Use of sugammadex in a patient with chronic kidney failure
}

\author{
Uso de sugamadex em paciente com insuficiência renal crônica
}

\author{
Gabriel Erazo Vaca (iD a , Christian Calderón Jaime (iD) ${ }^{a}$
}

a Servicio de Anestesiología, Hospital Luis Vernaza, GuayaquilEcuador.

\section{Correspondencia a:}

Gabriel Erazo-Vaca,

gabrielerazov@gmail.com

Recibido: 15 de octubre, 2020

Aceptado: 19 de noviembre, 2020 Publicado: 4 de enero, 2021

\section{CASO CLÍNICO}

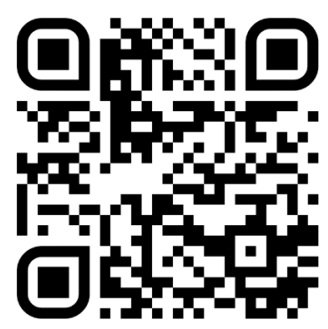

Escanea en tu dispositivo móvil o revisa este artículo en: https:// revistaclinicaguayaquil.org

\section{RESUMEN}

Los pacientes con enfermedad renal crónica poseen distintos tipos de factores, tanto fisiológicos como farmacológicos, que alteran la farmacocinética y farmacodinámica de los relajantes musculares no despolarizantes (RMND) lo que hace que la función neuromuscular sea impredecible. La Administración de Alimentos y Medicamentos de los Estados Unidos (FDA) determina como una contraindicación el uso del sugammadex en aquellos pacientes que presenten falla renal crónica asegurando que el aclaramiento de sugammadex se reduce y la vida media se prolonga en estos pacientes; es por este motivo la importancia de su estudio. En Ecuador, el sugammadex ingresó al Cuadro Nacional de Medicamentos Básicos en el año 2019 para determinados casos especiales. Presentamos el caso de un paciente masculino de 47 años con insuficiencia renal crónica en terapia restitutiva renal que fue sometido a una gastroduodeno anastomosis en Y de Roux más antrectomía laparoscópica, en quien se utilizó exitosamente sugammadex para reversión completa del bloqueo neuromuscular profundo.

Palabras clave: sugammadex; rocuronio; insuficiencia renal crónica; diálisis; reversión

\section{ABSTRACT \\ Patientswithchronickidneydiseasehavedifferenttypesofphysiological and pharmacological factors that alter the pharmacokinetics and pharmacodynamics of non-depolarizing muscle relaxants (NDMR), which make neuromuscular function unpredictable. The Food and Drug Administration (FDA) contraindicates to the use of sugammadex in those patients with chronic kidney disease, ensuring that the clearance of sugammadex is reduced and its half-life is prolonged in this group of patients; therefore, the importance of its study. In Ecuador, the sugammadex entered the national basic drug table in 2019 , only for certain cases. We present the case of a 47-year-old male with chronic kidney disease on renal replacement therapy who underwent gastroduodenal RouX-en-Y anastomosis plus laparoscopic antrectomy, in whom sugammadex was successfully used for complete reversal of deep neuromuscular blockade.}

Key words: sugammadex; rocuronium; chronic kidney disease; dialysis; reversal 


\section{RESUMO}

Os pacientes com doença renal crônica têm diferentes tipos de fatores, tanto fisiológicos como farmacológicos, que alteram a farmacocinética e a farmacodinâmica dos relaxantes musculares não-despolarizantes (NDMR) tornando a função neuromuscular imprevisível. A Administração de Alimentos e Drogas dos Estados Unidos (FDA) determina como contraindicação o uso de sugammadex naqueles pacientes que apresentam insuficiência renal crônica, assegurando que a liberação de sugammadex seja reduzida e que a meia-vida seja prolongada nestes pacientes; esta é a razão pela qual é importante estudá-la. No Equador, a sugammadex entrou na Lista Nacional de Drogas Básicas em 2019 para certos casos especiais. Apresentamos o caso de um paciente masculino de 47 anos de idade com insuficiência renal crônica submetido a terapia de substituição renal que foi submetido a uma anastomose gastroduodenal Roux-en-Y mais antrectomia laparoscópica, em que o sugammadex foi utilizado com sucesso para reversão completa do bloqueio neuromuscular profundo.

Palavras-chave: sugammadex; rocurônio; insuficiência renal crônica; diálise; reversão

\section{INTRODUCCIÓN}

Los pacientes con enfermedad renal crónica poseen distintos tipos de factores, fisiológicos y farmacológicos que alteran la farmacocinética y farmacodinámica de los relajantes musculares no despolarizantes (RMND) lo que hace que la función neuromuscular sea impredecible.

Los relajantes musculares, son fármacos que se utilizan en la anestesia general con la finalidad de facilitar la intubación orotraqueal y la ventilación mecánica, así como también, para optimizar las condiciones quirúrgicas al cirujano reduciendo el tono de la pared abdominal. Sin embargo, el proceso de recuperación del bloqueo neuromuscular residual puede llevar a múltiples complicaciones respiratorias durante el posoperatorio, estas incluyen desaturación de oxígeno, atelectasias y neumonía.

Con el objetivo de disminuir esos efectos, en Europa en el año 2008 se creó un nuevo medicamento denominado "Sugammadex" (Bridion, Merck \& Co, Whitehouse Stations, NJ) aprobado en diciembre del año 2015 por la Administración de Alimentos y Medicamentos de los Estados Unidos (FDA, por sus siglas en inglés, Food and Drug Administration). El sugammadex es una gamma-ciclodextrina modificada que se utiliza para revertir el bloqueo neuromuscular producido por los RMND, especialmente el rocuronio y el vecuronio, mediante la encapsulación en un estable complejo hidrosoluble, el cual es eliminado por vía renal; en pacientes con falla renal la vida media de eliminación del sugammadex y el rocuronio en 72 horas es de $29 \%$ y $4 \%$, respectivamente, versus $73 \%$ y $42 \%$ de los pacientes con función renal normal (1).

La FDA determina como una contraindicación el uso del sugammadex en aquellos pacientes que presenten falla renal crónica asegurando que el aclaramiento de sugammadex se reduce y la vida media se prolonga en estos pacientes; es por este motivo la importancia de estudiar los efectos de este fármaco en aquel grupo de pacientes. En Ecuador, el sugammadex ingresó al Cuadro Nacional de Medicamentos Básicos en el año 2019 únicamente para casos especiales, por lo que es indispensable su completo conocimiento para el manejo adecuado del fármaco (2-4).

Se presenta un caso y revisión de la literatura sobre la eficacia del sugammadex para reversión de los RMND en los pacientes con insuficiencia renal crónica que reciban terapia de restitución renal.

\section{DESCRIPCIÓN DEL CASO CLÍNICO}

Paciente masculino de 47 años con antecedentes patológicos personales de hipertensión arterial hace 6 años tratada con losartan $50 \mathrm{mg} /$ día, diabetes mellitus controlado con insulina glargina (Lantus $18 \mathrm{UI} /$ día) y enfermedad renal crónica en terapia sustitutiva renal trisemanal desde el año 2014 a través de una fístula arteriovenosa izquierda. No posee antecedentes quirúrgicos o alérgicos, riesgo quirúrgico cardiovascular moderado (Lee II/IV), riesgo de acuerdo a clasificación ASA III/V. El paciente fue programado para una gastroduodeno anastomosis en $Y$ de Roux más antrectomía laparoscópica.

Al examen físico, presentó un peso de $55 \mathrm{~kg}$, talla de $165 \mathrm{~cm}$, presión arterial 140/80 $\mathrm{mmHg}$, frecuencia cardiaca de $80 \mathrm{lpm}$, frecuencia respiratoria $16 \mathrm{rpm}, \mathrm{SpO}_{2}$ de $100 \%$ al aire ambiente. No hubo predictores de dificultad en la vía aérea, score de Mallampati II/IV, escala de Patil-Aldreti de $6 \mathrm{~cm}$, distancia interincisivos $>3 \mathrm{~cm}$. Además, no se identificó patología cardiopulmonar aparente.

Los análisis de laboratorio prequirúrgico reportan: hemoglobina $9 \mathrm{~g} / \mathrm{dl}$, hematocrito 
$26.3 \%$, plaquetas $258.000 / \mathrm{mm}^{3}$, creatinina 3.44 $\mathrm{mg} / \mathrm{dl}$, urea $43.1 \mathrm{mg} / \mathrm{dl}$, TP 15.7 segundos, INR 1.34, TTP 44.2 segundos, filtrado glomerular $23.82 \mathrm{ml} / \mathrm{min} / 1.73 \mathrm{~m}^{2}$, sin otros hallazgos relevantes; última hemodiálisis 24 horas previa cirugía.

Se realizó la monitorización con presión arterial no invasiva, pulsioximetría, electrocardiograma de tres electrodos, capnografía, y capnometría.

Previo a la inducción anestésica se preoxigena al paciente con oxígeno $3 \mathrm{l} / \mathrm{min}$ por tres minutos, luego se administra lidocaína 2\% S/E 35 mg bolo IV, fentanilo 75 ug IV, propofol $100 \mathrm{mg}$ IV y rocuronio $20 \mathrm{mg}(0.3 \mathrm{ug} / \mathrm{kg}) \mathrm{IV}$. Se procedió con la intubación orotraqueal, acoplándose a ventilación mecánica con parámetros protectivos.

Durante el transquirúrgico el paciente permaneció hemodinámicamente estable, el mantenimiento anestésico se manejó con sevoflurane CAM $1 \%$, remifentanilo $0.2 \mathrm{ug} / \mathrm{kg} /$ min. Se decide administrar cuatro bolos de 5 $\mathrm{mg}$ rocuronio $(0.07 \mathrm{mg} / \mathrm{kg})$ cada 30 minutos después del comienzo del procedimiento para mejorar las condiciones de la laparoscopía, la cual se mantiene estable hasta su finalización.

Treinta minutos antes de finalizar el procedimiento quirúrgico se procede a disminuir el $50 \%$ de la dosis del sevoflurane y del remifentanilo, 15 minutos después se decide cerrar el suministro de ambos, luego de 6 minutos se decide administrar sugammadex 200mg (3 mg/kg) IV para su reversión, siendo efectivo a los 4 minutos tras su administración, por lo que se extuba al paciente despierto, quien presentaba un buen patrón ventilatorio, respondía órdenes sencillas con movimientos musculares voluntarios. Luego, se lo observa por 15 minutos en quirófano no hallándose episodios de hipoventilación, ni de desaturación que hicieran sospechar la reaparición de relajación neuromuscular.

\section{DISCUSIÓN}

Durante la reversión espontánea, el rocuronio es tomado por el hígado y excretado a mayor medida por la vía biliar sin ser metabolizado; después de la reversión con sugammadex, el fármaco forma un complejo con el rocuronio el cual es excretado por intermedio del glomérulo a nivel renal. El sugammadex permite una reversión completa de un bloqueo neuromuscular profundo independientemente del tiempo quirúrgico; adicionalmente, la reversión con sugammadex ha demostrado ser más rápida y predecible, si lo comparamos con la neostigmina (5-6).

El sugammadex es una gamma-ciclodextrina que consiste en oligosacáridos enlazados alrededor de una cavidad central. Los relajantes musculares se envuelven rápidamente en la cavidad posterior a la administración del sugammadex, lo que los neutraliza, disminuyendo su nivel plasmático y creando un gradiente de concentración entre el plasma y la placa terminal neuromuscular. Este gradiente de concentración provoca el retiro del relajante muscular de la placa motora para dirigirse al plasma lo que conlleva a la neutralización del relajante muscular circundante; este mecanismo de acción explica el rápido efecto reversor del sugammadex (7).

El complejo sugammadex-rocuronio es extremadamente estable, por lo que la reversión neuromuscular del rocuronio por parte del sugammadex depende exclusivamente de la encapsulación e inactivación del rocuronio, y no de la excreción del complejo en la orina. En pacientes con insuficiencia renal crónica el complejo sugammadex-rocuronio es detectable en plasma hasta 7 días posterior a su administración. La eficiencia de la reversión realizada con sugammadex dependerá de la administración de la dosis adecuada para lograr neutralizar los relajantes musculares que se encuentren en la circulación (7).

A nivel mundial, aproximadamente 8.9 millones de personas se exponen al fármaco sin efectos adversos reportados, lo que demuestra su seguridad y eficacia. El sugammadex no posee los efectos muscarínicos no deseados de la neostigmina, sin embargo, existen estudios que muestran ciertos efectos a nivel del intervalo QT y los tiempos de coagulación. Adicionalmente, existen otros efectos adversos descritos, los cuales incluyen movimientos involuntarios en el $3 \%$ de los pacientes, disgeusia o parosmia en el $1 \%$, broncoespasmo en el $2.6 \%$, relajación residual relacionado a dosis subóptimas de sugammadex en un $1.3 \% \mathrm{y}$, raramente, reacciones alérgicas (5-7).

La parálisis residual (curarización) incrementa la morbilidad respiratoria postoperatoria; esta puede manifestarse como episodios de tos, atragantamiento o como dificultad en la capacidad de los pacientes para realizar respiraciones profundas. La recurarización ocurre con más frecuencia si se efectúa la reversión con neostigmina en comparación con 
el sugammadex (7).

El uso del sugammadex para la reversión de los bloqueos neuromusculares inducidos por el rocuronio en pacientes con falla renal es controversial, el efecto del bloqueo neuromuscular con dosis $0.6 \mathrm{mg} / \mathrm{kg}$ de rocuronio cuando se administra tras la inducción con propofol está reportado que es más prolongado en pacientes jóvenes y ancianos que tengan falla renal, comparado con pacientes que tengan función renal conservada (8).

La FDA determina que el uso de sugammadex no es recomendado para el uso de pacientes con falla renal severa, incluyendo a aquellos pacientes en plan de diálisis. Esto es debido a que el sugammadex es excretado en una molécula hidrosoluble por vía renal y no es posible su remoción con diálisis (excepto diálisis de alto flujo) $(2,3)$. Honing et al, detallan que el sugammadex no debería usarse en pacientes con un filtrado glomerular menor de $30 \mathrm{ml} / \mathrm{min}$ (7).

Un estudio en dos hospitales universitarios en el cual incluyeron 40 pacientes sometidos a trasplante renal, de los cuales 20 tenían enfermedad renal crónica con $\mathrm{ClCr}<30 \mathrm{ml} / \mathrm{min}$ y 20 pacientes del grupo control $\mathrm{ClCr}>90 \mathrm{ml} / \mathrm{min}$, los resultados demostraron que el uso de $4 \mathrm{mg} /$ $\mathrm{kg}$ de sugammadex es efectivo para la reversión de un bloqueo neuromuscular profundo, pero el proceso de recuperación es más lento cuando lo compararon con pacientes con función renal normal (9).

El bloqueo neuromuscular inducido por el rocuronio es prolongado en pacientes con falla renal debido a la reducción en su aclaramiento. El aclaramiento del rocuronio puede estar reducido en un $39 \%$ en pacientes con insuficiencia renal crónica comparado con pacientes con función renal conservada. Staals et al. investigaron la eficacia y la seguridad del sugammadex para la reversión del bloqueo neuromuscular inducido por el rocuronio en pacientes con insuficiencia renal crónica (aclaramiento creatinina $<30 \mathrm{ml} /$ min) comparado con pacientes con función renal normal (aclaramiento creatinina $>80 \mathrm{ml} /$ min); ellos determinaron que la administración de $2 \mathrm{mg} / \mathrm{kg}$ de sugammadex es efectivo para la reversión de un bloqueo neuromuscular moderado tanto para pacientes con insuficiencia renal crónica como para pacientes con función renal conservada (8).

Staals et al. identificaron que el uso para reversión de sugammadex $2 \mathrm{mg} / \mathrm{kg}$ es rápida y segura para pacientes con insuficiencia renal crónica, adicionalmente no mostraron pacientes con recurarización o complicación asociada a la reversión (8). Adicionalmente, Pfaff et al. demostraron en un paciente de 19 años con insuficiencia renal crónica que fue sometido a revisión de su derivación ventriculoperitoneal, resultando el uso de sugammadex seguro y eficaz (10).

Adams et al. realizaron un estudio observacional retrospectivo en el cual determinaron la eficacia y la seguridad del uso del sugammadex en pacientes con insuficiencia renal mediante la incidencia de re-intubación traqueal en las $48 \mathrm{~h}$ del postoperatorio. Identificaron 158 pacientes de los cuales el $14 \%$ se difirió su extubación debido a condiciones médicas pre-existentes o condiciones quirúrgicas. De los 136 pacientes restantes, 3 pacientes tuvieron que ser reintubados en las 48 horas del postoperatorio inmediato, debido a edema pulmonar por la sobrecarga volumétrica (2 pacientes) y empeoramiento de la sepsis (1 paciente). No identificaron incidencia de recurrencia en el bloqueo neuromuscular, además notaron que 24 pacientes (18\%) cuya reversión fue incompleta con el uso de neostigmina lograron una extubación exitosa tras la administración de sugammadex (3).

Por otra parte, Ezri et al. citan en su revisión el trabajo de Brueckmann B y col, en el que ningún paciente presentó parálisis residual después de la administración de sugammadex (0 de $74)$, comparado con el $43 \%$ después del uso de neostigmina (33 de 76 pacientes) $(11,12)$.

En el caso presentado podemos destacar la seguridadyeficacia del sugammadex, obteniendo los beneficios de un proceso de recuperación más lento, bloqueo neuromuscular menos prolongado, sin requerimiento de reintubación, ni inmediata (24 horas postoperatorio), ni mediata (hasta su egreso).

Se requieren estudios de mayor escala para establecer la seguridad de este fármaco y eventualmente considerar las condiciones que permitan su eso en esta población.

\section{AGRADECIMIENTOS}

Al Hospital Luis Vernaza, lugar donde se desarrolló el caso clínico.

\section{REFERENCIAS BIBLIOGRÁFICAS}

1. de Souza C, Tardelli M, Tedesco H, et al. Efficacy and safety of sugammadex in the reversal of deep 
neuromuscular blockade induced by rocuronium in patients with end-stage renal disease. Eur J Anaesthesiol 2015; 32:681-686. doi: 10.1097/ EJA.0000000000000312.

2. Weiskopf RB, James MF. Update of use of hydroxyethyl starches in surgery and trauma. J Trauma Acute Care Surg. 2015 Jun;78(6 Suppl 1):S54-9. doi: 10.1097/TA.0000000000000636.

3. Adams DR, Tollinche LE, Yeoh CB, Artman J, Mehta M, Phillips D, Fischer GW, Quinlan JJ, Sakai T. Shortterm safety and effectiveness of sugammadex for surgical patients with end-stage renal disease: a two-centre retrospective study. Anaesthesia. 2020 Mar;75(3):348-352. doi: 10.1111/anae.14914.

4. Lee HY, Jung KT. Advantages and pitfalls of clinical application of sugammadex. Anesth Pain Med (Seoul). 2020 Jul 31; 15(3):259-268. doi: 10.17085/ apm.19099.

5. Panhuizen IF, Gold SJ, Buerkle C, Snoeck MM, Harper NJ, Kaspers MJ, van den Heuvel MW, Hollmann MW. Efficacy, safety and pharmacokinetics of sugammadex $4 \mathrm{mg}$ kg-1 for reversal of deep neuromuscular blockade in patients with severe renal impairment. $\mathrm{Br} J$ Anaesth. 2015 May;114(5):777-84. doi: 10.1093/ bja/aet586.

6. Canakci E, Karatas A. The association between kidney function and efficacy of sugammadex in the reversal of neuromuscular blockade trainof-four and kidney functions. J Anesth Crit Care Open Access. 2018;10(5):188-196. doi: 10.15406/ jaccoa.2018.10.00387

7. Honing $G$, Martini $\mathrm{CH}$, Bom A, van Velzen $\mathrm{M}$, Niesters M, Aarts L, Dahan A, Boon M. Safety of sugammadex for reversal of neuromuscular block. Expert Opin Drug Saf. 2019 Oct;18(10):883891. doi: 10.1080/14740338.2019.1649393.
8. Staals LM, Snoeck MM, Driessen JJ, Flockton EA, Heeringa M, Hunter JM. Multicentre, parallelgroup, comparative trial evaluating the efficacy and safety of sugammadex in patients with endstage renal failure or normal renal function. $\mathrm{Br} J$ Anaesth. 2008 Oct;101(4):492-7. doi: 10.1093/bja/ aen216.

9. de Souza CM, Tardelli MA, Tedesco H, Garcia NN, Caparros MP, Alvarez-Gomez JA, de Oliveira Junior IS. Efficacy and safety of sugammadex in the reversal of deep neuromuscular blockade induced by rocuronium in patients with end-stage renal disease: A comparative prospective clinical trial. Eur J Anaesthesiol. 2015 Oct;32(10):681-6. doi: 10.1097/EJA.0000000000000312.

10. Pfaff K, Tumin D, Tobias JD. Sugammadex for Reversal of Neuromuscular Blockade in a Patient with Renal Failure. J Pediatr Pharmacol Ther. 2019 May-Jun;24(3):238-241. doi: 10.5863/1551-677624.3.238.

11. Ezri T, Boaz M, Sherman A, Armaly M, Berlovitz Y. Sugammadex: An Update. J Crit Care Med (Targu Mures). 2016 Feb 9;2(1):16-21. doi: 10.1515/jccm2016-0005.

12. Brueckmann B, Sasaki N, Grobara P, Li MK, Woo T, de Bie J, Maktabi M, Lee J, Kwo J, Pino R, Sabouri AS, McGovern F, Staehr-Rye AK, Eikermann M. Effects of sugammadex on incidence of postoperative residual neuromuscular blockade: a randomized, controlled study. $\mathrm{Br} J$ Anaesth. 2015 Nov; 115(5):743-51. doi: 10.1093/bja/aev104.

Conflicto de intereses. No se declara conflicto de intereses.

Fuente de financiamiento. Ninguna declarada por los autores.

\section{ACERCA DE LOS AUTORES}

1. Gabriel Erazo-Vaca. Médico posgradista del Servicio de Anestesiología, Hospital Luis Vernaza, Guayaquil Ecuador.

ORCID: 0000-0003-0161-0671

2. Christian Calderón-Jaime. Servicio de Anestesiología, Hospital Luis Vernaza, Ecuador.

ORCID: 0000-0002-4175-4250 